\title{
Association of selected factors with long-term prognosis and mortality after dual-chamber pacemaker implant
}

\author{
Maciej Dębski ${ }^{1}$, Mateusz Ulman ${ }^{1}$, Andrzej Ząbek ${ }^{1}$, Krzysztof Boczar ${ }^{1}$, \\ Kazimierz Haberka ${ }^{1}$, Marcin Kuniewicz ${ }^{1,2}$, Jacek Lelakowski ${ }^{1,3}$, Barbara Małecka ${ }^{1,3}$ \\ ${ }^{1}$ Department of Electrocardiology, John Paul II Hospital, Krakow, Poland \\ ${ }^{2}$ Department of Anatomy, Jagiellonian University Medical College, Krakow, Poland \\ ${ }^{3}$ Institute of Cardiology, Jagiellonian University Medical College, Krakow, Poland
}

\begin{abstract}
Background: Dual-chamber (DDD) pacing is the most widely utilised pacing modality in many parts of the world. The present study aimed to evaluate life expectancy of DDD pacemaker patients in comparison to the age- and sex-matched general population, assess changes in baseline characteristics over three decades of the inclusion period and determine the association between selected variables and patient survival.
\end{abstract}

Methods: This longitudinal study of consecutive de novo DDD pacemaker implantations performed between 1984 and 2014, with all-cause mortality until 2016 as the endpoint, was conducted at a singlecenter university hospital.

Results: Under assessment were 3928 patients with a total of 30,087 patient-years of survival time. Compared to the general population, the observed survival was significantly inferior until 12 years post $D D D$ pacemaker implant $(H R=1.499, p<0.001)$, whereas after 12 years of follow-up the observed survival was significantly superior $(H R=0.555, p<0.001)$. A comparison of patient baseline characteristics over three decades revealed the following significant changes: more elderly patients, more female patients, less patients with atrioventricular block, more patients with atrial fibrillation/atrial flutter $(A F / A F L)$ and fewer patients with an apical right ventricular (RV) lead position in the later decades. In multivariate analysis male sex and higher age were the only variables significantly associated with shorter survival time. Indication for pacing, history of pre-implant $A F / A F L, R V$ lead position and device infection were not associated with survival.

Conclusions: In the very-long-term follow-up of DDD pacemaker patients, the parameters associated with survival were sex and baseline age at first implantation. (Cardiol J 2019; 26, 6: 717-726)

Key words: mortality, survival, risk factors, cardiac pacing, atrial fibrillation

\section{Introduction}

Population-based observational studies and randomized controlled trials have assessed longterm survival and a variety of factors for their prognostic importance after pacemaker (PM) implant. Evaluated risk factors included baseline factors such as patient gender, age at implantation, decade of implantation, type of bradyarrhythmia, presence of atrial fibrillation (AF), cardiovascular diseases, non-cardiac comorbidities and periprocedural factors such as type of pacing mode, urgency of the procedure, position of the right ventricular (RV) lead and necessity of temporary pacing

Address for correspondence: Maciej Dębski, MD, Department of Electrocardiology, John Paul II Hospital, ul. Prądnicka 80, 31-202 Kraków, Poland, tel: +48 1261423 81, fax: +48 1263323 99, e-mail: maciekdebski@gmail.com 
[1-6]. Results have varied depending on population sample size, baseline characteristics, enrolment criteria, length of follow-up and the choice of evaluated factors. Currently, dual-chamber (DDD) pacing is by far the most widely utilized pacing modality in clinical practice in many parts of the world, and its use is exhibiting an increasing trend [7-9]. According to the 2013 European Society of Cardiology (ESC) guidelines, DDD pacing mode is the first choice in patients with sick sinus syndrome (SSS) and atrioventricular block (AVB) [10]. Despite its widespread use, the verylong-term survival of patients with DDD PMs has not been addressed separately from other pacing modalities in an analysis of an unselected, realworld cohort. Therefore, the present aim was to examine prognostic impact of selected variables on survival time and overall mortality of the DDD PM population compared to an age- and sex-matched population. Moreover, patient profiles and longterm survival outcomes were compared after DDD PM implantation across three successive decades at a single center.

\section{Methods}

The study cohort consisted of all consecutive patients who underwent de novo DDD PM implantation between 4 October 1984 (first DDD PM implantation) and 31 December 2014 at a highvolume, third-level reference university implantation center. Each patient was followed up after PM implantation up to 31 August 2016 or the time of death before 1 September 2016 . The data of patient survival status and deceased patient date of death were collected from the national death registration system. Information on death dates was available up until the end of August 2016. The endpoint was all-cause mortality. The data used in the analysis included (1) patient demographic baseline characteristics: date of birth, age at implantation and sex; (2) index arrhythmia (anti-bradycardia pacing indication): AVB defined as third-degree AVB, second-degree AVB and intraventricular conduction abnormalities (bundle branch block and/or fascicular block) with syncope or symptomatic SSS; (3) history of atrial fibrillation/atrial flutter (AF/ /AFL) prior to DDD PM implantation; (4) position of the RV lead: apical or non-apical at discharge from the department; (5) time of device-related infection onset and (6) date of death declared in the death certificate. This information was retrospectively gathered from paper and electronic medical records of hospitalizations when DDD PM implan- tation was performed, operative reports and outpatient pacemaker clinic records. If the patients had various coexisting types of bradyarrhythmia, the following priority ranking was applied for assigning the main indication for anti-bradycardia pacing: third-degree AVB, second-degree AVB, SSS and finally intraventricular conduction abnormality (bundle branch block and/or fascicular block) with syncope in case there was no other cause of syncope. SSS was represented by sinoatrial block, sinus node arrest, tachycardia-bradycardia syndrome and chronotropic incompetence. The term 'history of AF/AFL' was defined as AF and/or AFL documented on electrocardiogram prior to DDD PM implantation and included paroxysmal and persistent $\mathrm{AF}$ and/or AFL provided that the restoration of sinus rhythm was planned after DDD $\mathrm{PM}$ implantation. Patients with permanent AF/ /AFL were referred for VVI PMs throughout the study period. Device-related infection included local device infection and cardiac device-related infective endocarditis. The position of the RV lead was determined from operative reports and postprocedure, posteroanterior and left lateral chest radiographs.

Regarding the RV lead implantation technique, on a year-over-year basis, we specified the periods when RV apical lead fixation prevailed and when non-apical localizations were utilized more frequently. The implantation period 1984-2014 was divided into three successive time intervals referred to as decades: the first decade was from 1 October 1984 to 31 December 1994, the second was from 1 January 1995 to 31 December 2004 and the third was from 1 January 2005 to 31 December 2014. On a decade-over-decade basis, the number of patients, their baseline characteristics at the time of implantation and the type of RV lead position were compared.

Regarding survival data, the total duration between the first DDD PM implantation and either the date of death or end of the follow-up period (31 August 2016) was calculated for the whole cohort and referred to as patient-years of survival time. Additionally, life expectancy tables provided by Central Statistical Office for Poland for years 1990-2014 to match each person in the cohort with the age- and sex-matched life expectancy predicted at the year of DDD PM implantation were used [11]. Patients who underwent implantation between 1984 and 1989 were matched with the life expectancy predicted in 1990. The end date of follow-up which is 31 August 2016 was used to censor expected survival. 
Table 1. Comparison of the patient baseline characteristics across three successive decades of DDD pacemaker implantations.

\begin{tabular}{|c|c|c|c|c|c|}
\hline & $\begin{array}{c}\text { Total } \\
\text { population }\end{array}$ & $\begin{array}{c}1^{\text {st }} \text { decade } \\
\text { X } 1984-\text { XII } 1994\end{array}$ & $\begin{array}{c}2^{\text {nd }} \text { decade } \\
\text { | } 1995-\text { XII } 2004\end{array}$ & $\begin{array}{c}3^{\text {rd }} \text { decade } \\
\text { | } 2005-\text { XII } 2014\end{array}$ & $\mathbf{P}$ \\
\hline Number of patients & $3928(100 \%)$ & $210(5.3 \%)$ & $1144(29.1 \%)$ & $2574(65.5 \%)$ & \\
\hline Age [years]: & & & & & $<0.001$ \\
\hline $0-50$ & $290(7.4 \%)$ & $41(19.5 \%)$ & $135(11.8 \%)$ & $114(4.4 \%)$ & \\
\hline $51-70$ & $1537(39.1 \%)$ & $127(60.5 \%)$ & $531(46.4 \%)$ & $879(34.1 \%)$ & \\
\hline $71-80$ & $1498(38.1 \%)$ & $33(16.7 \%)$ & $400(35.0 \%)$ & $1065(41.4 \%)$ & \\
\hline $81-90$ & $576(14.7 \%)$ & $8(3.8 \%)$ & $76(6.6 \%)$ & $492(19.1 \%)$ & \\
\hline$>90$ & $27(0.7 \%)$ & $1(0.5 \%)$ & $2(0.2 \%)$ & $24(0.9 \%)$ & \\
\hline $\begin{array}{l}\text { Mean age at implantation } \\
\text { [years] }\end{array}$ & $69.8 \pm 12.1$ & $61.0 \pm 13.5$ & $66.5 \pm 12.0$ & $72.0 \pm 11.3$ & $<0.001$ \\
\hline Female sex & $1817(46.3 \%)$ & $88(41.9 \%)$ & $502(43.9 \%)$ & $1227(47.7 \%)$ & 0.045 \\
\hline Atrioventricular block & $1318(33.6 \%)$ & $113(53.8 \%)$ & $296(25.9 \%)$ & $909(35.3 \%)$ & - 0001 \\
\hline Sick sinus syndrome & $2610(66.4 \%)$ & $97(46.2 \%)$ & $848(74.1 \%)$ & $1665(64.7 \%)$ & 0.001 \\
\hline $\begin{array}{l}\text { History of atrial fibrillation/ } \\
\text { /atrial flutter }\end{array}$ & $1318(33.6 \%)$ & $22(10.5 \%)$ & $299(26.1 \%)$ & $997(38.7 \%)$ & $<0.001$ \\
\hline $\begin{array}{l}\text { Right ventricular lead apical } \\
\text { position }\end{array}$ & $1693(43.1 \%)$ & $210(100 \%)$ & $1107(96.8 \%)$ & $376(14.6 \%)$ & $<0.001$ \\
\hline
\end{tabular}

\section{Statistical analysis}

The data were evaluated using IBM SPSS Statistics for Windows, Version 25 (IBM Corp., Armonk, NY, USA). Normality was tested using Shapiro-Wilk test for samples less than or equal to 2000 and Kolmogorov-Smirnov test for samples greater than 2000 . Continuous variables are expressed as mean, standard deviation and additionally as median and interquartile range (IQR) for variables with non-normal distribution. Groups were compared using the $\chi^{2}$ test for discrete variables and the Mann-Whitney U test for continuous variables with non-normal distribution. Event-free rates were calculated using the Kaplan-Meier analysis method and compared using the log-rank test. The associations between patient survival and the selected variables were assessed using a Cox proportional hazards model and presented as hazard ratio (HR) with $95 \%$ confidence interval (CI). The associations between patient survival and variables with timevarying effect (strength of a factor was not constant over time) and time-varying covariates (value of the factor was not constant over time) were tested using Cox model with time-dependent covariate. A p value of $<0.05$ was considered statistically significant.

\section{Results}

A total of 3932 consecutive patients underwent primary DDD PM implantation during the study pe- riod. The data of patient survival status on the last day of follow-up were available for 3928 (99.9\%) patients. Four $(0.1 \%)$ patients were excluded from the analysis due to unverified survival status because of unavailability of an identification number. Notably, for all baseline variables considered in the study population (3928 patients), there was no missing data.

The patient baseline clinical characteristics and a comparison of data from successive decades of DDD PM implantation are presented in Table 1. The mean age at the time of implantation was $69.8 \pm 12.1$, median (IQR) 71.9(14.2), range 15.3-96.6 years; $53.7 \%$ of patients were male; $2610(66.4 \%)$ patients had SSS; 1318 (33.6\%) patients had AVB defined as third-degree AVB (552 patients), second-degree AVB (737 patients) and bundle branch block with syncope (28 patients). AF/AFL before implantation was observed in 1318 (33.6\%) patients. Women were older than men at the time of implantation: $70.5 \pm 11.7$, median (IQR) 72.6 (13.1) vs. $69.2 \pm 12.4$, median (IQR) 71.2 (14.8) years $(\mathrm{p}<0.001)$; patients with SSS were older than those with AVB: $70.4 \pm 11.2$, median (IQR) 72.2 (13.2) vs. $68.6 \pm 13.7$, median (IQR) 71.3 (16.1) years $(\mathrm{p}=0.004)$; and patients with a history of AF/AFL were older than AF/AFL-free patients: $71.9 \pm 10.1$, median (IQR) 73.0 (12.0) vs. $68.7 \pm 12.9$, median (IQR) 71.2 (15.2) years $(\mathrm{p}<0.001)$. AVB was present in $25.6 \%$ of women 
and $44.4 \%$ of men $(\mathrm{p}<0.001)$. The prevalence of AF/AFL before implantation reached $40.9 \%$ in women and $27.2 \%$ in men ( $<<0.001)$. From 1984 to 2005 , the majority of RV leads were placed at the RV apex, and from 2006, the majority of $\mathrm{RV}$ leads were placed in a non-apical position. A comparison of baseline characteristics among the three successive decades revealed a significant rise in number of procedures, average age at implantation, number of women referred for DDD PM, prevalence of SSS and prevalence of AF/ /AFL prior to implantation (Table 1). Furthermore, stratification of patients by age group disclosed a significant decreasing trend in the proportion of patients before or in the seventh decade of life and an increasing trend in the proportion of patients in eighth, ninth and tenth decades of life $(\mathrm{p}<0.001)$.

A total of 30,087 patient-years of survival time was calculated for 3928 patients. The mean observation time was $7.7 \pm 5.3$, median (IQR) $6.4(6.7)$ years. During the follow-up period 1435 (36.5\%) patients died. The mean age of the deceased patients was $79.9 \pm 9.7$, median (IQR) 81.6 (11.3) years. The Kaplan-Meier estimates for survival probability after DDD PM implantation at 1, 2, 5, 10,15 and 20 years after the procedure amounted to $96 \%, 92 \%, 82 \%, 62 \%, 46 \%$ and $32 \%$, respectively. With regard to age- and sex-matched survival data, the predicted number of deaths amounted to $1262(32.1 \%)$ and predicted mean observation time was $8.4 \pm 4.6$, median (IQR) 7.4 (5.8) years. The expected survival curve had a reverse sigmoidal shape and crossed the observed survival at 12 years after implantation. Until 12 years of follow-up the observed risk of death was higher than expected ( $\mathrm{HR}=1.499,95 \%$ CI 1.376-1.633, $\mathrm{p}<0.001)$, whereas after 12 years observed mortality was lower than expected $(\mathrm{HR}=0.555,95 \%$ CI 0.468-0.658, p < 0.001) (Fig. 1). The Kaplan-Meier curves revealed no significant difference in survival with regard to index arrhythmia (AVB vs. SSS; $\mathrm{p}=0.92$ ) (Fig. 2A) and a history of AF/AFL before implantation ( $\mathrm{p}=0.503$ ) (Fig. 2B). Male sex was associated with unfavourable survival ( $p<0.001$ ) (Fig. 2C). Patients with apical RV lead position compared to patients with non-apical lead position had a significantly better survival during the first 10 years after implantation $(p=0.002)$ (Fig. 3A). With regard to the time of implantation, the later the decade of implantation the worse survival was observed with statistically significant linear trend for factor levels ( $\mathrm{p}<0.001$ ) (Fig. 4A). However, after survival adjustment for sex and age at implantation the difference in survival between

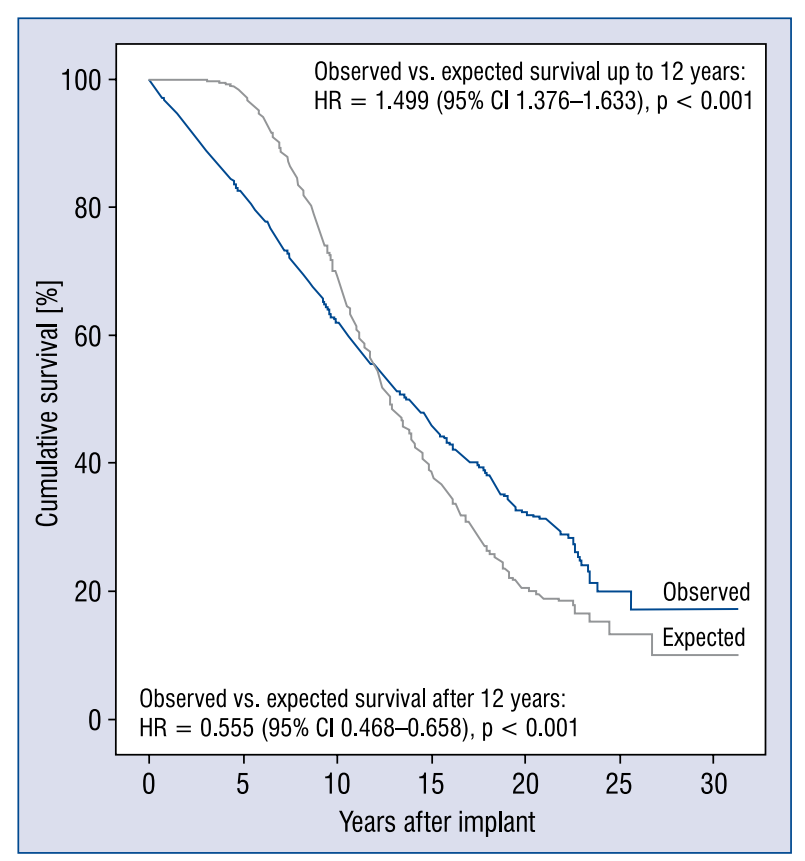

Figure 1. Survival of patients with DDD pacemaker relative to the age- and sex-matched general population. $\mathrm{HR}$ - hazard ratio; $\mathrm{Cl}$ - confidence interval.

apical and non-apical RV lead position group was attenuated $(p=0.196)$ (Fig. 3B). Furthermore, survival during 10 years after implantation after adjustment to sex and age was superior in patients with implantation in third decade compared to patients with implantation in second decade $(\mathrm{p}=$ $=0.017$ ). Comparing sex- and age-adjusted survival curves for first vs. second decade and first vs. third decade there were no statistically significant differences (Fig. 4B).

The Cox proportional hazard regression model demonstrated that older age at implantation and male sex were significantly associated with higher mortality. By contrast, pacing indication and a history of AF/AFL were not associated with survival (Fig. 5).

During follow-up $43(1.1 \%)$ patients, 20 females, developed device-related infection after a mean follow-up of $7.3 \pm 5.3$, median (IQR) 7.2 (8.7) years. Twenty-six (60.4\%) patients with local infections were observed and 17 (39.5\%) patients with cardiac-device related infective endocarditis. Within 1 year from implantation $6(14 \%)$ patients developed device-related infections. Death occurred in $14(32.6 \%)$ patients, 5 females, after a mean period of $9.2 \pm 6.0$, median (IQR) 8.4 (6.8) years from infection diagnosis. Device-related infection was not associated with an increased risk of death $(\mathrm{HR}=0.693,95 \%$ CI 0.097-4.93, $\mathrm{p}=0.714)$. 


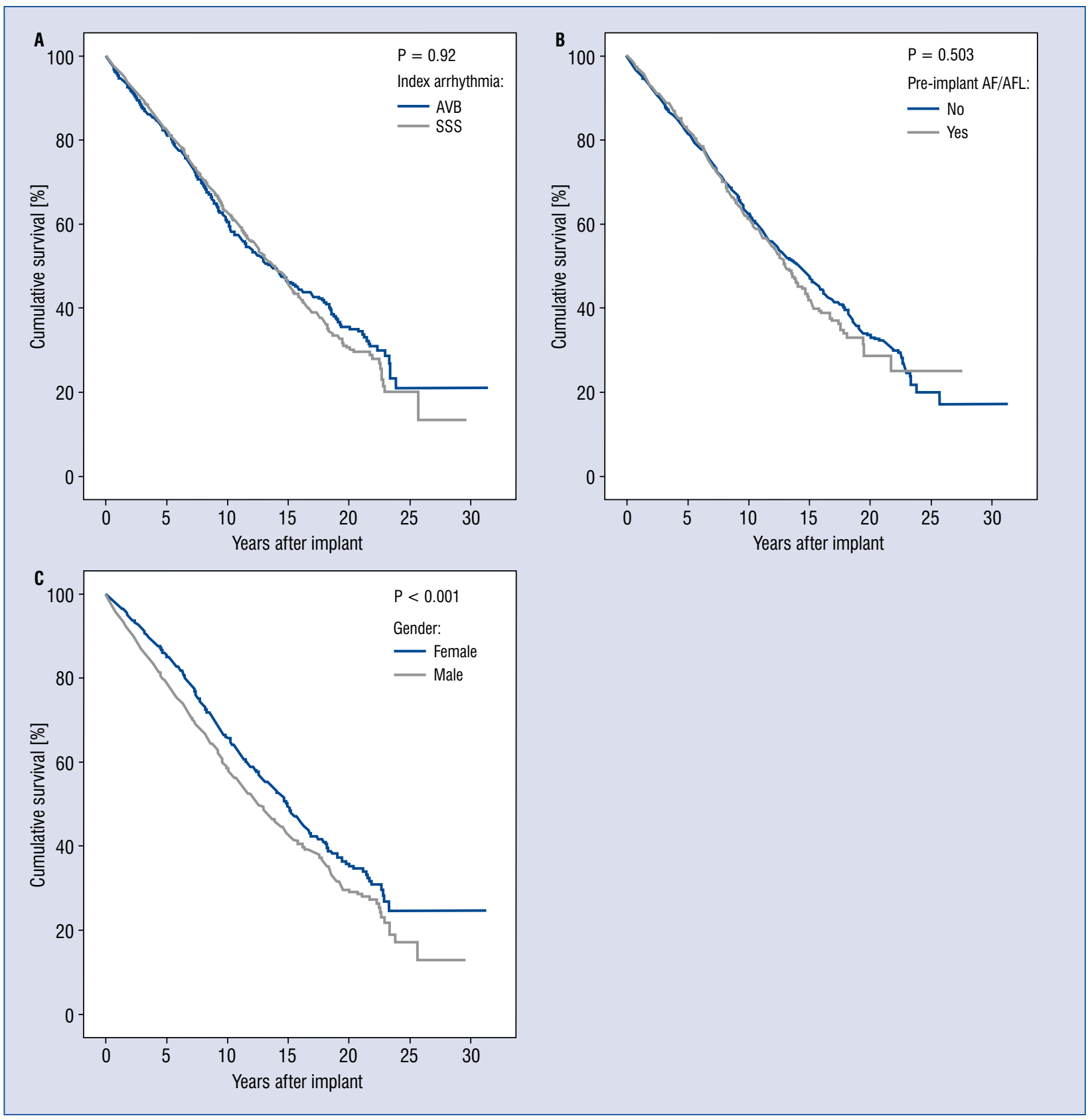

Figure 2. Survival of patients with atrioventricular block (AVB) relative to patients with sick sinus syndrome (SSS) (A), survival of patients with a history of atrial fibrillation/atrial flutter (AF/AFL) relative to patients without pre-implant AF/AFL (B), survival of women relative to men (C).

\section{Discussion}

The long-term survival of PM patients has been assessed in several population-based studies of general PM populations that included from 1.5\% to $73.3 \%$ patients with DDD PM $[1,2,4,5,12-14]$. Importantly, no study has analysed mortality in very-long-term DDD PM patients only, or identified independent risk factors for mortality in this population. Therefore, the present study, which had an excellent $(99.9 \%)$ rate of complete data on overall survival, was designed to allow comparisons in a large group of consecutive patients enrolled without exclusion criteria who received a DDD PM at a single center and were free from permanent $\mathrm{AF} / \mathrm{AFL}$ at the moment of implantation. With 3928 patients, 30,087 patient-years of survival time and an observation time of three decades, this is one of 


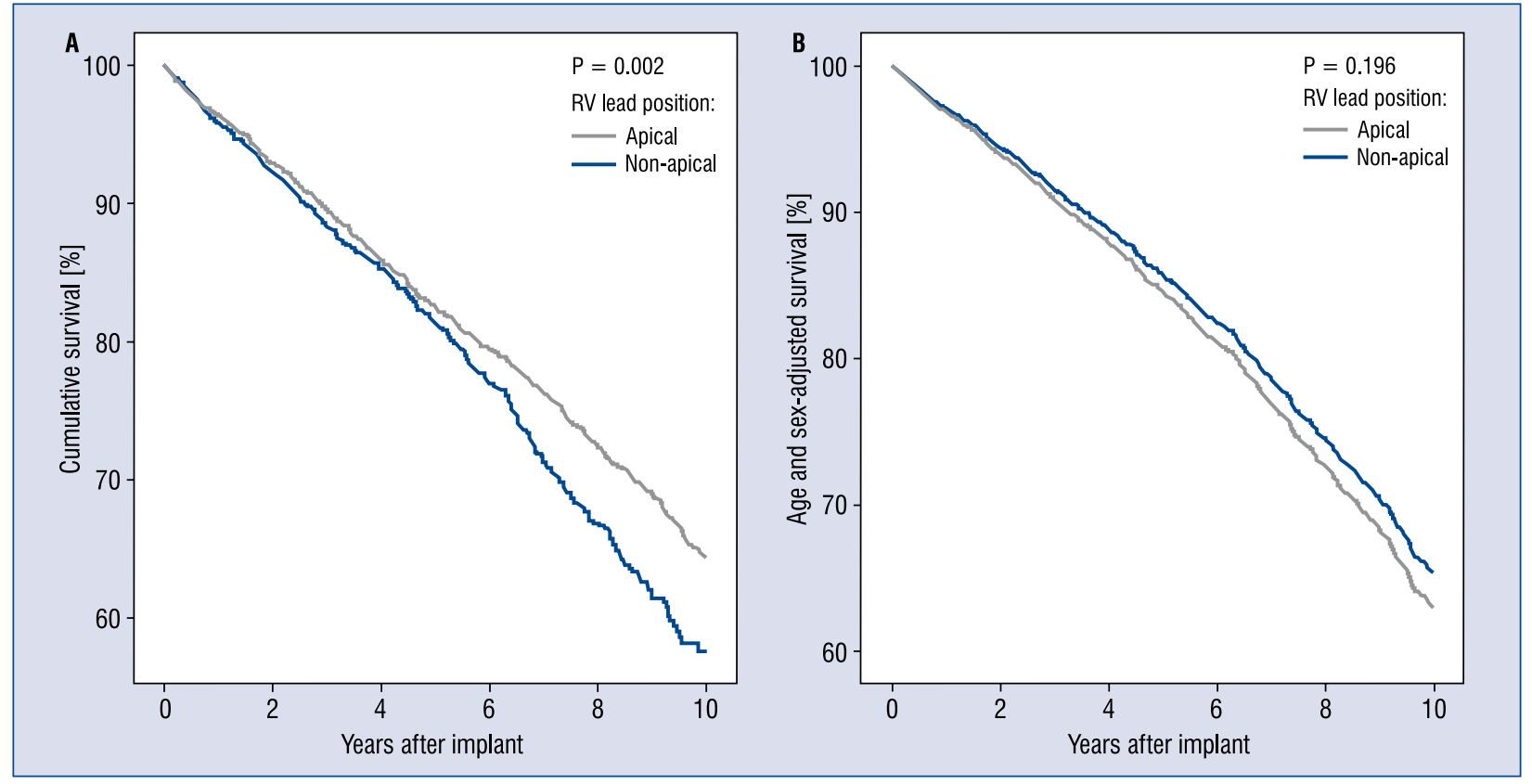

Figure 3. Survival during 10 years after implantation in patients with right ventricular (RV) lead in apical position relative to patients with RV lead in non-apical position (A), survival after adjustment for age and sex (B).

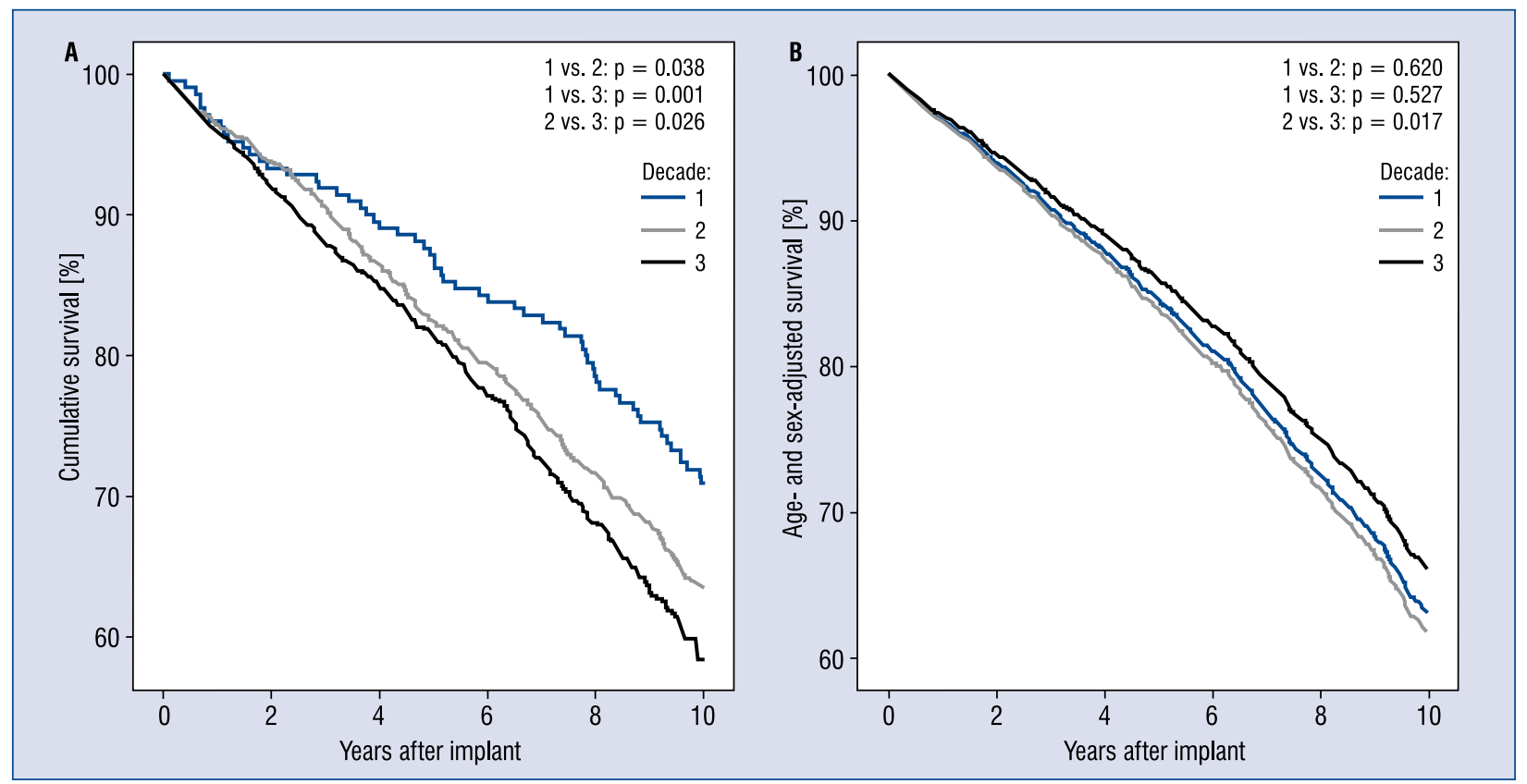

Figure 4. Survival during 10 years after implantation according to the decade of implantation (A), survival after adjustment for age and sex (B).

the largest studies to reliably examine very-long-term survival in patients referred for primary DDD PM implantation.

In reports on survival of a PM population, authors have concluded that prognosis of PM recipients without significant comorbidities at baseline approached that of the general population $[1,5,13,14]$. Among the factors contributing most to increased mortality in the PM group relative to the control population were significant non-cardiac 


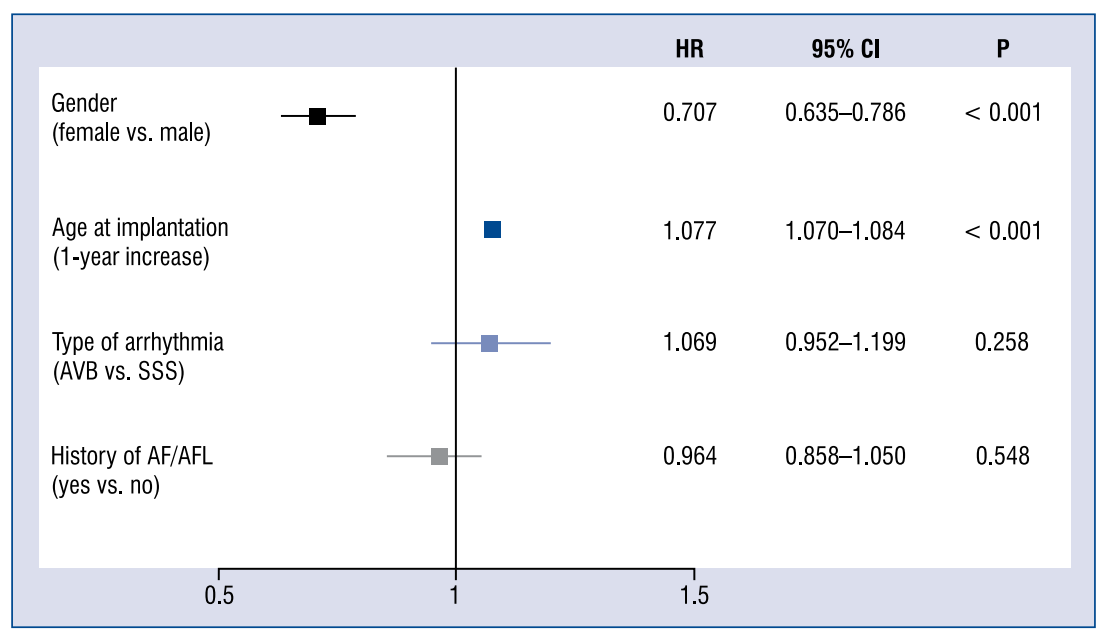

Figure 5. Factors associated with survival in multivariable Cox regression model; AVB - atrioventricular block; SSS - sick sinus syndrome; AF/AFL — atrial fibrillation/atrial flutter; $\mathrm{HR}$ - hazard ratio; $\mathrm{Cl}$ - confidence interval.

comorbidities and structural heart disease $[1,5$, 13, 14]. Pyatt et al. [4] have reported significantly higher overall mortality in PM cohort compared to expected mortality during a period of 8 years after implantation. The present data, showing significantly worse overall survival in DDD cohort relative to the expected survival until 12 years after implantation are in agreement with the results of Pyatt et al. [4]. On the other hand, after 12 years post-implant survival among DDD recipients significantly exceeded survival of general population. Presumably, long-term benefit from DDD PM beyond 12 years of follow-up might have applied predominantly to a population without significant comorbidities and were relatively young at baseline. Reasons for improved survival after 12 years, post-implant in DDD PM patients, might have included prevention against sudden bradyarrhythmic death and regular follow-up with cardiologist which might have allowed early recognition and treatment of cardiovascular diseases.

The 1-year survival rate of the present DDD cohort was $96 \%$. These data appear to accord with 1-year overall survival rates from $90.5 \%$ to $96 \%$ as provided in reports of DDD populations [4, 12, $15,16]$ and rates from $91 \%$ to $94 \%$ as provided in reports of general PM populations $[1,5,13$, 14]. At 5-year follow-up, cumulative survival rate was $82 \%$. Reported 5-year survival rate of DDD cohorts was significantly lower and accounted for $58 \%$ to $64.7 \%$ [4, 12, 17], whereas in general PM cohorts, this value reached $58.2 \%$ to $69 \%$ $[2,5,18]$. Long-term estimated survival probability at 10 -year follow-up after implantation was $62 \%$, which is broadly consistent with other reports of general PM populations: from $44.8 \%$ to $75.4 \%$ $[2,13]$. The 20 -year survival probability was estimated at $32 \%$ in the present study compared with $21.4 \%$ observed by Brunner et al. [2]. Importantly, in the study Brunner et al. [2], a significant number of patients (38.6\%) were lost to follow-up and censored as alive on the day of their last visit, which renders their information on estimated survival rates less accurate.

Regarding baseline characteristics, a higher prevalence of men across the study period was observed, which is in accordance with the majority of studies in DDD PM populations [15-17, 19] and general PM populations [1, 2, 5, 9, 12, 13, 18] except for Scandinavian populations, in which the prevalence of women receiving first DDD PM was reported to be higher than that of men [20, $21]$. In the present study, the prevalence of men exhibited a statistically significant decreasing trend from $58 \%$ in the first decade to $52 \%$ in the third decade, which is opposed to observations of a stable proportion of men in successive eras of PM implantation [1,2]. The age of patients at first PM implantation increased with each decade, similar to a trend observed in western countries $[2,7,18]$. Furthermore, the present study identified a significant increase in PM utilization among older patients ( $>70$ years). In countries with advanced health systems, the percentage of PM recipients older than 80 years was $>30 \%$ and exhibited a significantly increasing trend $[1,8,9$, 18]. Female patients were older than men at the time of implantation, as it has been observed in a majority of countries [1,2, 8, 22], and were more likely to present with SSS $[1,2,22]$. There has 
been a shift in the main indications, with AVB being more prevalent in 1984-1994 and the domination of SSS in 1995-2014. Unlike the results herein, a higher incidence of high-grade AVB than of SSS throughout the study period has been frequently reported in general PM populations $[1,2,4,5,9$, $12-14,18]$. Importantly, in the present study, the prevalence of pre-implant AF/AFL soared across the study period, reaching $39 \%$ in the third decade, a trend that can probably be attributed to enhanced detection of $\mathrm{AF} / \mathrm{AFL}$, increasing age of patients [23] and shift of indications towards SSS [20, 24].

The significant association between male sex and older age at baseline and worse survival has been noted previously $[1,2,4,5,13]$ and corresponds to the fact that women generally have a longer life expectancy. As expected, the present study demonstrated that in the Cox regression model, age and male sex were independently associated with mortality after primary DDD implantation. For each additional year of age at implantation, a $7.8 \%$ increase in mean risk of death was observed; in the general PM population, this value has been reported as $5 \%$ to $9 \%[2,4,5,12]$. By contrast, no significant difference in survival with regard to either index arrhythmia or a history of AF/AFL was detected in the present study. The literature on the influence of index arrhythmia on survival comprises conflicting results. In a multivariate analysis of the study population, Brunner et al. [2] observed that SSS was associated with better survival than was AVB; however, on considering patients with first implantation during the last decade (1991-2000), this effect was no longer significant. Furthermore, Jahangir et al. [12] and Pyatt et al. [4] have identified that AVB is a risk factor for increased mortality compared with SSS [4, 12]. Conversely, Udo et al. [5], Mayosi et al. [14] and Jelić et al. [13] have demonstrated that survival of SSS and AVB patients is comparable.

In the FOLLOWPACE study, a history of atrial tachyarrhythmia was not an independent predictor of survival [5]. Conversely, Bradshaw et al. [1] demonstrated that a history of AF was significantly associated with reduced 1-year and 5-year survival. Of note, with increasing patient age at implantation across the second and third decades, age- and sex-adjusted survival of patients displayed an improving trend. The available data indicate that either the later the first implantation occurs in the study period, the better the prognosis of the PM recipient [2] or that there is no association between the era of implantation and mortality [1]. Regarding the RV lead position, after adjustment for sex and age at implantation there was no significant association with mortality. Witt et al. [6] assessed 3450 unselected patients who underwent DDD PM implantation between 2004 and 2014, among whom the RV lead was positioned at the RV apex in the majority of patients $(71.9 \%)$ and less commonly at the septum $(6.9 \%)$ or other RV regions (21.2\%). Authors reported that an apical RV lead position was associated with increased mortality compared with a septal position group (31\% vs. $24 \%, \mathrm{p}=0.02)$. Patients with very high levels of pacing, greater than $90 \%$, had a significantly lower mortality rate in the septal pacing group (16\% vs. $31 \%, p=0.03)$, whereas patients with very low levels of pacing, less than $10 \%$, did not have a significant difference in mortality ( $13 \%$ vs. $23 \%$, $\mathrm{p}=0.10$ ) [6]. Due to the retrospective design of the present study and the findings of Witt et al. [6], none of the aforementioned results can be taken as a definitive answer to the long-debated question of whether an apical position of the RV pacing lead is worse than a non-apical position.

In the present study, an infection rate of $1.1 \%$ per patient was observed, which is in line with previous reports from literature. Hercé et al. [25] in a study based on a registry which included 2496 patients observed $35(1.4 \%)$ cases with devicerelated infections. Greenspon et al. [26] reported that the rate of device-associated infections in the United States rose from $1.53 \%$ in 2004 to $2.41 \%$ in 2008, likely due to an increase of patients with multiple comorbidities. Earlier reports on DDD PM population with implantation between 1984 and 2002 showed the rate of device-related infection was less than or equal to $1.2 \%[27,28]$. The present study shows that device-related infection was not a risk factor for increased mortality during follow-up and patients diagnosed with pacing system infection had relatively good long-term survival. Results herein, are in keeping with the findings of a prospective matched cohort-study of Deharo et al. [29] who observed no significant excess in all-cause long-term mortality in infection cohort compared with controls without devicerelated infection.

\section{Limitations of the study}

The main limitation is the retrospective nature of this study, with all its inherent limitations. First, data regarding other baseline factors that possibly influenced survival, such as a history of concomitant diseases, medications, functional status (New York Heart Association class) and urgency of the procedure (elective/emergency), were not com- 
plete for the whole population and were therefore not included in the analysis. Similarly, the percentage of RV pacing was not available for all patients, therefore the association between RV lead position and mortality could not be further analyzed in subgroups with different requirements of RV pacing. Second, the prevalence of pre-implant AF/AFL in the first two decades may have been underrated due to lower awareness and surveillance. Third, selection bias of presumably 'sicker' patients with AVB referred for single-chamber ventricular pacing could not be excluded and presumably 'healthier' patients with SSS to single-chamber atrial pacing because evidence supporting the use of DDD systems as a first-choice pacing mode for both indications was unavailable at the time.

\section{Conclusions}

With an increasing number of DDD PM implantations over time, a significant change in patient baseline characteristics was observed: average age at implantation continued to rise, more women were referred for implantation and the prevalence of $\mathrm{AF} / \mathrm{AFL}$ prior to implantation grew rapidly. During 12 years after implantation, survival of the DDD cohort was significantly worse than in an age- and sex-matched general population, however, after 12 years the survival of DDD recipients were significantly better than expected. Male sex and age were the only clinical variables associated with a shortened survival time and an increased probability of death. Indication for pacing, history of pre-implant AF/AFL, RV lead position and device-related infection were not associated with survival.

\section{Conflict of interest: None declared}

\section{References}

1. Bradshaw PJ, Stobie P, Knuiman MW, et al. Life expectancy after implantation of a first cardiac permanent pacemaker (1995-2008): A population-based study. Int J Cardiol. 2015; 190: 42-46, doi: 10.1016/j.ijcard.2015.04.099, indexed in Pubmed: 25912118.

2. Brunner M, Olschewski M, Geibel A, et al. Long-term survival after pacemaker implantation. Prognostic importance of gender and baseline patient characteristics. Eur Heart J. 2004; 25(1): 88-95, indexed in Pubmed: 14683747.

3. Flaker G, Greenspon A, Tardiff B, et al. Death in patients with permanent pacemakers for sick sinus syndrome. Am Heart J. 2003; 146(5): 887-893, doi: 10.1016/S0002-8703(03)00429-0, indexed in Pubmed: 14597940.

4. Pyatt JR, Somauroo JD, Jackson M, et al. Long-term survival after permanent pacemaker implantation: analysis of predictors for increased mortality. Europace. 2002; 4(2): 113-119, indexed in Pubmed: 12135241.

5. Udo EO, van Hemel NM, Zuithoff NPA, et al. Prognosis of the bradycardia pacemaker recipient assessed at first implantation: a nationwide cohort study. Heart. 2013; 99(21): 1573-1578, doi: 10.1136/heartjnl-2013-304445, indexed in Pubmed: 23969476.

6. Witt CM, Lenz CJ, Shih HH, et al. Right ventricular pacemaker lead position is associated with differences in long-term outcomes and complications. J Cardiovasc Electrophysiol. 2017; 28(8): 924-930, doi: 10.1111/jce.13256, indexed in Pubmed: 28543771 .

7. Greenspon AJ, Patel JD, Lau E, et al. Trends in permanent pacemaker implantation in the United States from 1993 to 2009: increasing complexity of patients and procedures. J Am Coll Cardiol. 2012; 60(16): 1540-1545, doi: 10.1016/j.jacc.2012.07.017, indexed in Pubmed: 22999727.

8. Mond HG, Proclemer A. The 11th world survey of cardiac pacing and implantable cardioverter-defibrillators: calendar year 2009--a World Society of Arrhythmia's project. Pacing Clin Electrophysiol. 2011; 34(8): 1013-1027, doi: 10.1111/j.15408159.2011.03150.x, indexed in Pubmed: 21707667.

9. Proclemer A, Ghidina M, Gregori D, et al. Trend of the main clinical characteristics and pacing modality in patients treated by pacemaker: data from the Italian Pacemaker Registry for the quinquennium 2003-07. Europace. 2010; 12(2): 202-209, doi: 10.1093/europace/eup346, indexed in Pubmed: 19903671.

10. Brignole M, Auricchio A, Baron-Esquivias G, et al. 2013 ESC guidelines on cardiac pacing and cardiac resynchronization therapy. Europace. 2013; 15: 1070-1118.

11. https://stat.gov.pl/en/topics/population/life-expectancy/life-expectancy-in-poland,1,3.html.

12. Jahangir A, Shen WK, Neubauer SA, et al. Relation between mode of pacing and long-term survival in the very elderly. J Am Coll Cardiol. 1999; 33(5): 1208-1216, indexed in Pubmed: 10193718.

13. Jelić V, Belkić K, Djordjević M, et al. Survival in 1,431 pacemaker patients: prognostic factors and comparison with the general population. Pacing Clin Electrophysiol. 1992; 15(2): 141-147, indexed in Pubmed: 1372412.

14. Mayosi BM, Little F, Millar RN. Long-term survival after permanent pacemaker implantation in young adults: 30 year experience. Pacing Clin Electrophysiol. 1999; 22(3): 407-412, indexed in Pubmed: 10192849.

15. Connolly SJ, Kerr CR, Gent M, et al. Effects of physiologic pacing versus ventricular pacing on the risk of stroke and death due to cardiovascular causes. Canadian Trial of Physiologic Pacing Investigators. N Engl J Med. 2000; 342(19): 1385-1391, doi: 10.1056/NEJM200005113421902, indexed in Pubmed: 10805823.

16. Liao JN, Chao TF, Tuan TC, et al. Long-term outcome in patients receiving permanent pacemaker implantation for atrioventricular block: Comparison of VDD and DDD pacing. Medicine (Baltimore). 2016; 95(35): e4668, doi: 10.1097/MD.0000000000004668, indexed in Pubmed: 27583889.

17. Toff WD, Camm AJ, Skehan JD. Single-chamber versus dualchamber pacing for high-grade atrioventricular block. N Engl J Med. 2005; 353(2): 145-155, doi: 10.1056/NEJMoa042283, indexed in Pubmed: 16014884.

18. Uslan DZ, Tleyjeh IM, Baddour LM, et al. Temporal trends in permanent pacemaker implantation: a population-based study. Am 
Heart J. 2008; 155(5): 896-903, doi: 10.1016/j.ahj.2007.12.022, indexed in Pubmed: 18440339.

19. Marchandise S, Scavée C, le Polain de Waroux JB, et al. Long-term follow-up of DDD and VDD pacing: a prospective nonrandomized single-centre comparison of patients with symptomatic atrioventricular block. Europace. 2012; 14(4): 496-501, doi: 10.1093/europace/eur345, indexed in Pubmed: 22071380.

20. Nielsen JC, Thomsen PE, Højberg S, et al. DANPACE Investigators. A comparison of single-lead atrial pacing with dual-chamber pacing in sick sinus syndrome. Eur Heart J. 2011; 32(6): 686-696, doi: 10.1093/eurheartj/ehr022, indexed in Pubmed: 21300730.

21. Fored CM, Granath F, Gadler F, et al. Atrial vs. dual-chamber cardiac pacing in sinus node disease: a register-based cohort study. Europace. 2008; 10(7): 825-831, doi: 10.1093/europace/eun118, indexed in Pubmed: 18467299.

22. Nowak B, Misselwitz B, Erdogan A, et al. Do gender differences exist in pacemaker implantation? Results of an obligatory external quality control program. Europace. 2010; 12(2): 210-215, doi: 10.1093/europace/eup312, indexed in Pubmed: 19864309.

23. Schnabel RB, Yin X, Gona P, et al. 50 year trends in atrial fibrillation prevalence, incidence, risk factors, and mortality in the Framingham Heart Study: a cohort study. Lancet. 2015; 386(9989): 154-162, doi: 10.1016/S0140-6736(14)61774-8, indexed in Pubmed: 25960110.
24. Lamas G, Lee K, Sweeney M, et al. Ventricular pacing or dualchamber pacing for sinus-node dysfunction. N Engl J Med. 2002; 346(24): 1854-1862, doi: 10.1056/nejmoa013040.

25. Hercé B, Nazeyrollas P, Lesaffre F, et al. Risk factors for infection of implantable cardiac devices: data from a registry of 2496 patients. Europace. 2013; 15(1): 66-70, doi: 10.1093/europace/ eus284, indexed in Pubmed: 23097224.

26. Greenspon AJ, Patel JD, Lau E, et al. 16-year trends in the infection burden for pacemakers and implantable cardioverterdefibrillators in the United States 1993 to 2008. J Am Coll Cardiol. 2011; 58(10): 1001-1006, doi: 10.1016/j.jacc.2011.04.033, indexed in Pubmed: 21867833.

27. Dębski M, Ulman M, Ząbek A, et al. Gender differences in dual-chamber pacemaker implantation indications and longterm outcomes. Acta Cardiol. 2016; 71(1): 41-45, doi: 10.2143/ AC.71.1.3132096, indexed in Pubmed: 26853252.

28. Ulman M, Dębski M, Ząbek A, et al. Long-term follow-up of DDD pacing mode. Kardiol Pol. 2014; 72(6): 519-526, doi: 10.5603/ KP.a2014.0020, indexed in Pubmed: 24526555.

29. Deharo JC, Quatre A, Mancini J, et al. Long-term outcomes following infection of cardiac implantable electronic devices: a prospective matched cohort study. Heart. 2012; 98(9): 724-731, doi: 10.1136/heartjnl-2012-301627, indexed in Pubmed: 22523057. 